Sensitivity analysis for pattern mixture models

Peer-reviewed author version

Curran, Desmond; MOLENBERGHS, Geert; THIJS, Herbert \& VERBEKE, Geert (2003) Sensitivity analysis for pattern mixture models. In: Journal of

Biopharmaceutical Statistics, 14. p. 125-143.

DOI: $10.1081 / \mathrm{BIP}-120028510$

Handle: http://hdl.handle.net/1942/433 


\title{
Sensitivity Analysis for Pattern Mixture Models.
}

\author{
Desmond Curran, Geert Molenberghs, Herbert Thijs and Geert Verbeke
}

May 23, 2003

\begin{abstract}
Incomplete series of data is a common feature in quality of life studies, in particular in chronic diseases where attrition of patients is high. Two alternative approaches to modeling longitudinal data with incomplete measurements have frequently been proposed in the literature, selection models and pattern-mixture models. In this paper we focus on, by way of sensitivity analysis, extrapolating incomplete patterns using identifying restrictions. Perhaps the best known ones are so-called complete case missing value restrictions (CCMV), where, for a given pattern, the conditional distribution of the missing data, given the observed data, is equated to its counterpart in the completers. Available case missing value (ACMV) restrictions equate this conditional density to the one calculated from the subgroup of all patterns for which all required components have been observed. Neighboring case missing value restrictions (NCMV) equate this conditional density to the one calculated from the the pattern with one additional measurement obtained. In this paper, these three identifying restriction strategies are used to multiply impute missing data in a study in metastatic prostate cancer. Multiple imputation is employed to reduce the uncertainty of single imputation. It is shown how hypothesis testing and sensitivity analyses are carried out in this setting.
\end{abstract}

\section{Introduction}

One of the major complications in the analyses of quality of life (QL) data is that of missing data. In particular drop out may be problematic in QL studics since it is likcly that paticnts with the poorest QL scores dropout earlier, especially in cancer clinical trials in patients with 
advanced disease (Hopwood et, al, 1994, Curran et, al 1998). A way of describing the various mechanisms that govern incompleteness is provided by Rubin's paradigm. Rubin (1976) described three missing data mechanisms: missing completely at random (MCAR, dropout is independent of observed and unobserved scores), missing at random (MAR, dropout is independent of unobserved scores but dependent on observed scores) and missing not at random (MNAR, dropout is dependent on at least one unobserved score).

MAR appears to be a sensible 'middle ground' when dealing with incomplete data. However, one has to consider the possibility of a MNAR dropout mechanism. Two alternative approaches to modeling longitudinal data with incomplete measurements are selection models (Diggle and Kenward 1994) and pattern-mixture models (Little 1993, 1995, Hogan and Laird 1987). Thesc modclling frameworks approach the issuc of dropout in two distinct ways: in selection models the dropout probability is conditional on the measurement process, whereas in pattern-mixture models the measurement model is conditional on the dropout pattern. Although selection and pattern-mixture models are interchangeable from a probabilistic point of view, since they represent different factorizations of the same joint distribution, in practice they encourage different kinds of simplifying assumptions. In selection models, the likelihood for both the dropout model and measurement models are maximized simultancously resulting in a maximized joint likclihood during which some assumptions are made which are not fundamentally testable, e.g., the dependence of the dropout process on measurements which have not been obtained.

In selection models, all patients contribute to the model, through their likelihood contribution, at all time points whether they have dropped out or not. However, for pattern-mixture models patients only contribute to parameter estimates prior to dropout. Pattern-mixture models are underidentified (Glynn, Laird, and Rubin 1986). Consequently, Little (1993, 1994, 1995) suggested the use of so-called identifying restrictions to overcome this underidentification: inestimable parameters of the incomplete patterns are set equal to (functions 
of) the parameters describing the distribution of the completers. Little (1993) shows how these constraints can be used to identify all parameters in the model and so obtain estimates for these and the marginal probabilities. For example, complete case missing value (CCMV) restrictions (Little 1993) essentially equate conditional distributions beyond time $t$, i.e., those unidentifiable from this dropout group, to the same conditional distributions from the completers. While some authors perceive this under-identification as a drawback, we believe it can be an asset since it forces one to reflect on the assumptions made. In a $\mathrm{QL}$ study we may wish to answer two scientific questions: (1) what is the difference with respect to QL between the two treatment groups while patients remain on-study (progression-free)? or (2) what is the difference with respect to QL between the two treatment groups while patients remain alive? To answer these questions we may need to extrapolate our results and to do so with caution and under a number of different assumptions. This may be done using identifying restrictions.

QL data in longitudinal studies may be missing for a variety of reasons including progression of disease, treatment toxicity or patient refusal. However, most methods of analysis focus on a single dropout mechanism and do not take into account multiple reasons for dropout or patterns of missing data. Using the identifying restriction strategy to impute missing data, thus cxtrapolating incompletc pattcrns, we can incorporatc both the rcasons for missingness and the patterns of missingness into the imputation process. Employing several identifying restrictions allows us to perform a sensitivity analysis, thus addressing the uncertainty caused by dropout. This paper further explores these concepts and illustrates how this may be used in the context of a quality of life analysis in prostate cancer. Missing data patterns are extended using identifying restrictions and the method of conditional mean imputation.

Multiple imputation is employed to reduce the uncertainty of single imputation. The concept of multiple imputation refers to replacing each missing value with more than one imputed value. 'The goal is to combine the simplicity of imputation strategies, with unbiasedness in 
both point estimates and measures of precision. Some simple imputation procedures may yield inconsistent point estimates as soon as the missingness mechanism surpasses MCAR. This could be overcome to a large extent with conditional mean imputation, but the problem of underestimating the variability of the estimators is common to all methods since they all treat imputed values as observed values. By imputing several values for a single missing component, this uncertainty is explicitly acknowledged.

\section{The Data}

EORTC trial 30903 was designed as a prospective multicenter randomized phase III study comparing flutamide versus prednisone in hormone resistant metastatic prostate cancer patients. The main endpoint of the trial was survival. Flutamide and prednisone were administered daily until progression whereafter patients were treated according to the investigators' discretion. Progression was defined as either: an increase in pain score by $\geq 1$ category; an increase in daily analgesic dose by $\geq 25 \%$; any need to give additional anti-pain treatment, e.g. radiotherapy; deterioration of WHO performance status by $\geq 1$ category. Quality of life should have been evaluated at randomization, 3 and 6 weeks later, and at subsequent six-weekly intervals. Although, it was not clearly defined in the protocol most institutions did not perform QL assessments after progression. The EORTC QLQ-C30 (Aaronson et al 1993) was used to assess QL. In this report, we focus on the Global health status/QL scale of the EORTC QLQ-C30. The scale scores were constructed using the standard procedures recommended by the EORTC Quality of Life Study Group (Fayers et al 1999), i.e., scores were calculated by averaging items within scales and transforming average scores linearly to a 0 to 100 scale, with higher scores representing a better QL. Further details on the clinical analysis and the QL analysis are described elsewhere (Fossa et al 1999).

Between January 1992 and March 1998, 201 patients were entered into EORTC trial 30903 
(101 patients were randomized into the prednisone treatment arm and 100 into the flutamide treatment arm). Figure 1 presents a Kaplan-Meier plot of progression-free survival and a table of QL assessment compliance. The median duration of progression free survival was 3.4 and 2.3 months in the prednisone and flutamide arms, respectively. The main reason for patients going off-study was progression or death. As may be seen in Figure 1, the attrition of patients is substantial in both treatment arms. For this reason, only the assessments up until 24 weeks were used in this analysis. Table 1 shows the various missingness patterns. Twenty patients completed QL questionnaires at all five assessment time points. Monotone dropout patterns (i.e., a complete series of questionnaires before dropout) were observed in 104 cases. Intermittent missing questionnaires was also a problem with 46 patients having exactly 1 missing questionnaire and the remaining 11 patients having more than 1 missing questionnaire in a series before dropout.

As the main objective was to investigate (a) differences between treatment groups during treatment and (b) change from baseline it was decided to analyze change scores from baseline, i.e., observed scores minus baseline scores. This transformation resulted in response scores which were approximately normally distributed and more continuous in mature than the original QL score.

As illustrated by Curran et al (2002) it is useful to explore longitudinal QL data using graphical techniques before advancing to model fitting. Initially we plotted the response variable against time by treatment group and dropout pattern (see Figure 2). The dropout patterns were defined based on the dropout times, i.e., 3, 6, 12, 18, 24 and >24 weeks. However, as only 16 and 18 patients dropped out at week 24 and 30, respectively, patterns 4 and 5 were collapsed into a single pattern. From Figure 2 it appears that the scores in the prednisone arm increase from baseline to 3 weeks, but tend to decrease just before dropout suggesting that dropout is not completely at random. In contrast, the mean scores in the flutamide arm show very little change during the treatment period. 


\section{Pattern-Mixture Models and MAR}

The missing data taxonomy is usually presented in the selection modeling framework rather than in the pattern-mixture context. Here, we show that pattern-mixture models can be classified similarly, and further that the intermediate MAR category is connected to particular kinds of restrictions on the parameters of a pattern-mixture model in the case of monotone missingness. We will now show how pattern-mixture models can be classified using exactly the same taxonomy as is used for selection models. Furthermore, we establish a link between this classification and the identifying restrictions proposed in Little (1993). Clearly, selection models and pattern-mixture models coincide under the MCAR assumption as in both cases the joint distribution of the measurements and the missing data mechanism reduces to a distribution of the measurements and a distribution for the missing data mechanism, i.e. using the classical taxonomy $f(\boldsymbol{y}, d)=f(\boldsymbol{y}) f(d)$.

Next, we show that MAR can be expressed in a pattern-mixture framework through so-called available case missing value (ACMV) restrictions. Little's CCMV restrictions set a conditional density of unobserved components given a particular set of observed components equal to the corresponding conditional density in the subgroup of completers. ACMV restrictions equate this conditional density to the one calculated from the subgroup of all patterns for which all required components have been observed.

Assume a complete measurement sequence is of length $n$. Recall that the classical taxonomy considers the structure of $f(d \mid \boldsymbol{y})$. The missing data are MAR if a subject's missingness mechanism depends on its observed outcomes only, $f\left(d=t+1 \mid y_{1}, \ldots, y_{n}\right)=f(d=t+$ $\left.1 \mid y_{1}, \ldots, y_{t}\right)$, for $t=1, \ldots, n$.

In our setting of longitudinal data with dropouts, CCMV can be defined formally as the 
condition that for each $t \geq 2$ and for $j<t$ :

$$
f\left(y_{t} \mid y_{1}, \ldots, y_{t-1}, d=j+1\right)=f\left(y_{t} \mid y_{1}, \ldots, y_{t-1}, d=n+1\right),
$$

whereas ACMV is the condition that for all $t \geq 2$ and $j<t$ :

$$
f\left(y_{t} \mid y_{1}, \ldots, y_{t-1}, d=j+1\right)=f\left(y_{t} \mid y_{1}, \ldots, y_{t-1}, d>t\right) .
$$

If there are only 2 time points ( $n=2$ ), then ACMV and CCMV coincide. Based on equation (1), Molenberghs et al (1998) have shown that, for longitudinal data with dropouts, MAR is equivalent to ACMV. Details regarding the practical consequences of this equivalence of this can be found in Thijs et al (2002). Kenward, Molenberghs, and Thijs (2003) have shown how a set of restrictions can be constructed where dropout can depend on the current, possible unobserved measurement, but not on future measurements.

The restrictions discussed here will be incorporated in strategies to fit pattern-mixture models in Section 5.

\section{PMM, Sensitivity Analysis, and Identifying Restric- tions}

Sensitivity analysis for pattern-mixture models can be conceived in many different ways. Crucial aspects are whether pattern-mixture and selection modeling are to be contrasted with one another or whether the pattern-mixture modeling is the central focus of interest. It is natural as well to conduct sensitivity analysis within the pattern-mixture family. The

key area where sensitivity analysis should be focused is on the unidentified components of the model and the way(s) in which this is handled.

Little $(1993,1994)$ advocated the usc of identifiying restrictions and prescntcd a number of examples. We will outline a general framework for identifying restrictions in Section 4, with 
CCMV (introduced by Little 1993), ACMV, and neighboring case missing value restrictions (NCMV) as important special cases. Since ACMV is the natural counterpart of MAR in the PMM framework, this provides a way to compare ignorable selection models with their counterpart in the pattern-mixture setting. Michiels, Molenberghs, and Lipsitz (1999) took up this idea in the context of binary outcomes, with a marginal global odds ratio model to describe the measurement process (Molenberghs and Lesaffre 1994).

We restrict attention to monotone patterns. In general, let us assume we have $t=1, \ldots, T$ dropout patterns where the dropout indicator is $d=t+1$. For pattern $t$, the complete data density is given by

$$
f_{t}\left(y_{1}, \ldots, y_{T}\right)=f_{t}\left(y_{1}, \ldots, y_{t}\right) f_{t}\left(y_{t+1}, \ldots, y_{T} \mid y_{1}, \ldots, y_{t}\right)
$$

The first factor is clearly identified from the observed data, while the second factor is not. It is assumed that the first factor is known or, more realistically, modeled using the observed data. Then, identifying restrictions are applied in order to identify the second component.

While, in principle, completely arbitrary restrictions can be used by means of any valid density function over the appropriate support, strategies which relate back to the observed data deserve privileged interest. One can base identification on all patterns for which a given component, $y_{s}$ say, is identified. A general expression for this is

$$
f_{t}\left(y_{s} \mid y_{1}, \ldots y_{s-1}\right)=\sum_{j=s}^{T} \omega_{s j} f_{j}\left(y_{s} \mid y_{1}, \ldots y_{s-1}\right), \quad s=t+1, \ldots, T .
$$

We will use $\boldsymbol{\omega}_{s}$ as shorthand for the set of $\omega_{s j}$ 's used. Every $\boldsymbol{\omega}_{s}$ which sums to one provides a valid identification scheme.

Let us incorporate (3) into (2):

$$
f_{t}\left(y_{1}, \ldots, y_{T}\right)=f_{t}\left(y_{1}, \ldots, y_{t}\right) \prod_{s=0}^{T-t-1}\left[\sum_{j=T-s}^{T} \omega_{T-s, j} f_{j}\left(y_{T-s} \mid y_{1}, \ldots, y_{T-s-1}\right)\right]
$$


Expression (4) clearly shows which information is used to complement the observed data density in pattern $t$ in order to establish the complete data density.

Let us consider three special but important cases. Little (1993) proposes CCMV which uses the following identification:

$$
f_{t}\left(y_{s} \mid y_{1}, \ldots y_{s-1}\right)=f_{T}\left(y_{s} \mid y_{1}, \ldots y_{s-1}\right), \quad s=t+1, \ldots, T .
$$

In other words, information which is unavailable is always borrowed from the completers.

Alternatively, the nearest identified pattern can be used:

$$
f_{t}\left(y_{s} \mid y_{1}, \ldots y_{s-1}\right)=f_{s}\left(y_{s} \mid y_{1}, \ldots y_{s-1}\right), \quad s=t+1, \ldots, T \text {. }
$$

We will refer to these restrictions as neighboring case missing values or NCMV.

The third special case of (3) will be ACMV of which the definition is presented in (1). Thus, ACMV is reserved for the counterpart of MAR in the PMM context. Let us derive the corresponding $\boldsymbol{\omega}_{s}$ vectors. Expression (3) can be restated as

$$
f_{t}\left(y_{s} \mid y_{1}, \ldots, y_{s-1}\right)=f_{(\geq s)}\left(y_{s} \mid y_{1}, \ldots, y_{s-1}\right),
$$

for $s=t+1, \ldots, T$. Here, $f_{(\geq s)}(. \mid.) \equiv f(. \mid ., d>s)$, with $d$ an indicator for time of dropout, which is one more than the length of the observed sequence. Now, we can transform (7) as follows:

$$
\begin{aligned}
f_{t}\left(y_{s} \mid y_{1}, \ldots, y_{s-1}\right) & =f_{(\geq s)}\left(y_{s} \mid y_{1}, \ldots, y_{s-1}\right) \\
& =\sum_{j=s}^{T} \frac{\alpha_{j} f_{j}\left(y_{1}, \ldots, y_{s-1}\right)}{\sum_{j=s}^{T} \alpha_{j} f_{j}\left(y_{1}, \ldots, y_{s-1}\right)} f_{j}\left(y_{s} \mid y_{1}, \ldots, y_{s-1}\right) .
\end{aligned}
$$

Next, comparing (8) to (3) yields:

$$
\omega_{s j}=\frac{\alpha_{j} f_{j}\left(y_{1}, \ldots, y_{s-1}\right)}{\sum_{\ell=s}^{T} \alpha_{\ell} f_{\ell}\left(y_{1}, \ldots, y_{s-1}\right)} .
$$


We have now derived two equivalent explicit expressions of (1). Expression (8) is the conditional density of a mixture, whereas (3) with (9) is a mixture of conditional densities. Clearly, $\boldsymbol{\omega}$ defined by (9) consists of components which are nonnegative and sum to one. In other words, a valid density function is defined.

Restrictions (3), with the CCMV, NCMV, and ACMV forms as special cases, can be incorporated in a comprehensive strategy to fit pattern-mixture models.

\subsection{Special Case: 3 Measurements}

To illustrate the ideas outlined above in general, we focus on the special case where there are only three patterns and identification (4) takes the following form:

$$
\begin{aligned}
f_{3}\left(y_{1}, y_{2}, y_{3}\right)= & f_{3}\left(y_{1}, y_{2}, y_{3}\right) \\
f_{2}\left(y_{1}, y_{2}, y_{3}\right)= & f_{2}\left(y_{1}, y_{2}\right) f_{3}\left(y_{3} \mid y_{1}, y_{2}\right), \\
f_{1}\left(y_{1}, y_{2}, y_{3}\right)= & f_{1}\left(y_{1}\right)\left[\omega f_{2}\left(y_{2} \mid y_{1}\right)+(1-\omega) f_{3}\left(y_{2} \mid y_{1}\right)\right] \\
& \times f_{3}\left(y_{3} \mid y_{1}, y_{2}\right) .
\end{aligned}
$$

Since $f_{3}\left(y_{1}, y_{2}, y_{3}\right)$ is completely identifiable from the data, and for $f_{2}\left(y_{1}, y_{2}, y_{3}\right)$ there is only one possible identification, given (3), the only place where a choice has to be made is for pattern 1. Setting $\omega=1$ corresponds to NCMV, while $\omega=0$ implies CCMV. Using (9) in this particular case, ACMV corresponds to

$$
\omega=\frac{\alpha_{2} f_{2}\left(y_{1}\right)}{\alpha_{2} f_{2}\left(y_{1}\right)+\alpha_{3} f_{3}\left(y_{1}\right)} .
$$

The conditional density $f_{1}\left(y_{2} \mid y_{1}\right)$ in (12) can be rewritten as

$$
f_{1}\left(y_{2} \mid y_{1}\right)=\frac{\alpha_{2} f_{2}\left(y_{1}, y_{2}\right)+\alpha_{3} f_{3}\left(y_{1}, y_{2}\right)}{\alpha_{2} f_{2}\left(y_{1}\right)+\alpha_{3} f_{3}\left(y_{1}\right)} .
$$




\subsection{Imputation and Analysis Strategy Outline}

To apply PMM modeling with identifying restrictions to practical situations, the following strategy can be followed.

1. Fit a model to the pattern-specific identifiable densities: $f_{t}\left(y_{1}, \ldots, y_{t}\right)$. This results in a parameter estimate, $\hat{\gamma}_{t}$.

2. Select an identification method of choice.

3. Using this identification method, determine the conditional distributions of the unobserved outcomes, given the observed ones:

$$
f_{t}\left(y_{t+1}, \ldots, y_{T} \mid y_{1}, \ldots, y_{t}\right)
$$

4. Using the methodology outlined in Rubin (1987), draw multiple imputations for the unobserved components, given the observed outcomes and the correct pattern-specific density (14).

5. Analyze the multiply-imputed sets of data using the method of choice. This can be another pattern-mixture model, but also a selection model or any other desired model.

6. Inferences can be conducted using an $F$ reference distribution as proposed by Li, Raghunathan, and Rubin (1991).

Earlier, we have seen how general identifying restrictions (3), with CCMV, NCMV, and ACMV as special cases, lead to the conditional densities for the unobserved components, given the observed ones. This came down to deriving expressions for $\boldsymbol{\omega}$, such as in (9) for ACMV. This endeavor corresponds to items 2 and 3 of the strategy outline (4.2). In order to carry out item 4, we need to draw imputations from these conditional densities. 
Let us proceed by studying the special case of three measurements first. To this end, we consider identification scheme (10)-(12) and we start off by avoiding the specification of a parametric form for these densities. The following steps are required:

1. Estimate the parameters of the identifiable densities: $f_{3}\left(y_{1}, y_{2}, y_{3}\right), f_{2}\left(y_{1}, y_{2}\right)$, and $f_{1}\left(y_{1}\right)$. Then, for each of the $m$ imputations, we have to execute the following steps.

2. Draw from the parameter vectors as in the first step of the strategy. It will be assumed that in all densities from which we draw, this parameter vector is used.

3. For pattern 2. Given an observation in this pattern, with observed values $\left(y_{1}, y_{2}\right)$, calculate the conditional density $f_{3}\left(y_{3} \mid y_{1}, y_{2}\right)$ and draw from it.

4. For pattern 1. We now have to distinguish three substeps.

(a) Given $y_{1}$, and the proportions $\alpha_{2}$ and $\alpha_{3}$ of observations in the second and third patterns, respectively, determine $\omega$. Every $\omega$ in the unit interval is valid. Special cases are:

- For NCMV, $\omega=1$.

- For CCMV, $\omega=0$.

- For ACMV, $\omega$ is calculated from (13). Note that, given $y_{1}$, this is a constant. Generate a random uniform variate, $U$ say. (Note that, strictly speaking, this draw is unnecessary for the boundary NCMV and CCMV cases.)

(b) If $U \leq \omega$, calculate $f_{2}\left(y_{2} \mid y_{1}\right)$ and draw from it. Otherwise, do the same based on $f_{3}\left(y_{2} \mid y_{1}\right)$.

(c) Given the observed $y_{1}$ and given $y_{2}$ which has just been drawn, calculate the conditional density $f_{3}\left(y_{3} \mid y_{1}, y_{2}\right)$ and draw from it. 
All steps but the first one have to be repeated $M$ times, to obtain the same number of imputed datasets. Inference then proceeds as outlined in Rubin (1987) and Li, Raghunathan, and Rubin (1991).

When the observed densities are estimated using linear mixed models, $f_{3}\left(y_{1}, y_{2}, y_{3}\right), f_{2}\left(y_{1}, y_{2}\right)$, and $f_{1}\left(y_{1}\right)$ produce fixed-effect and variance parameters. Let us group all of them in $\gamma$ and assume a draw is made from their distribution, $\gamma^{*}$ say. To this end, their precision estimates need to be computed.

Let us illustrate this procedure for (11). Let us assume that the $i$ th subject has only two measurements, and hence belongs to the second pattern. Let its design matrices be $X_{i}$ and $Z_{i}$ for the fixed effects and random effects, respectively. Its mean and variance for the third pattern are:

$$
\begin{aligned}
\boldsymbol{\mu}_{i}(3) & =X_{i} \boldsymbol{\beta}^{*}(3) \\
V_{i}(3) & =Z_{i} D^{*}(3) Z_{i}^{\prime}+\Sigma_{i}(3)
\end{aligned}
$$

where (3) indicates that the parameters are specific to the third pattern.

Now based on (15)-(16), and the observed values $y_{i}=\left(y_{i 1}, y_{i 2}\right)^{\prime}$, the parameters for the conditional density follow immediately:

$$
\begin{aligned}
\boldsymbol{\mu}_{i, 2 \mid 1}(3) & =\boldsymbol{\mu}_{i, 2}(3)+V_{i, 21}(3)\left[V_{i, 11}(3)\right]^{-1}\left(\boldsymbol{y}_{i}-\boldsymbol{\mu}_{i, 2}(3)\right) \\
V_{i, 2 \mid 1}(3) & =V_{i, 22}(3)-V_{i, 21}(3)\left[V_{i, 11}(3)\right]^{-1} V_{i, 12}(3)
\end{aligned}
$$

where a subscript 1 indicates the first two components and a subscript 2 refers to the third component. Draws from each conditional density are entirely similar.

In several cases, the conditional density is a mixture of normal densities. Then, drawing from (3) consists of two steps: 
- Draw a random uniform variate $U$ to determine which of the $n-s+1$ components one is going to draw from. Specifically, the $k$ th component is chosen if

$$
\sum_{j=s}^{k-1} \omega_{s j} \leq U<\sum_{j=s}^{k} \omega_{s j},
$$

where $k=s, \ldots, n$. Note that if $k=1$, the left hand sum is set equal to zero.

- Draw from the $k$ th component.

\section{Analysis of QL Data}

In order to illustrate the methodology developed for identifying restrictions we will apply it to the analysis of longitudinal QL data. We will fit a pattern-mixture model to the observed data and use standard model simplification techniques to obtain a final model. This will be compared with the final models obtained using the identifying restrictions in Section 5.3. Note that this is an important advantage of the proposed methodology. Not only does it allow to estimate the parameters and standard errors of a model under given restrictions but in addition classical model simplification can be conducted. Indeed, starting from an elaborate model for the quality of life scores and maintaining the restrictions, one may want to reduce the model so that it only contains sufficiently significant effects.

\subsection{Pattern Mixture Model Fitted to Observed Data}

Several baseline clinical variables were considered as covariates in the model. These included demographic variables: age, WHO performance status and pain assessed by the clinician. Several models were fitted starting with a complex model which included a 3-way-interaction term (time-treatment-pattern) in the means model and a variance-covariance structure which included an autogressive order $1, \mathrm{AR}(1)$, and a residual variance, ascribed to instantaneous replication. The final model selected, which was the simplest model consistent with the data, 
consisted of baseline covariates and an interaction between time and treatment for the mean model while the variance-covariance was defined as a pattern specific AR(1) structure, with in addition a variance associated with measurement error.

\subsection{Fitting Models to the Imputed Data}

As was described in Section 5.1 a separate model was fitted within each pattern. The resulting parameter estimates and their estimated asymptotic covariance matrices were used to extrapolate the patterns as described in Section 4.2 .

The initial multiple imputation results are presented graphically. Figure 3 shows the mean response profiles for the multiply imputed datasets using the identifying restrictions CCMV, ACMV and NCMV. For patterns 1 to 4 there is some variability in the estimated profiles across the three restrictions. Using the CCMV identifying restriction, results in an increase in both treatment arms in pattern 1, whereas using ACMV and NCMV restrictions, results in the scores in the flutamide arm remaining approximately the same over time while in the prednisone arm they tend to decrease towards the fourth and fifth assessment. Roughly speaking, CCMV extrapolates rather towards a rise whereas NCMV seems to predict more of a decline. Further, ACMV predominantly indicates a constant mean score over time. This observation needs to be considered carefully. Since these patients drop out mainly because they progress, a rise in QL seems unlikely. Hence, it is possible that the dropout mechanism is not CCMV, since this strategy always refers to the 'best' group, in the sense that it groups patients who stay longer in the study and hence have on average a better prognosis. ACMV, which compromises between all strategies may be more realistic, but NCMV may be even better since information is borrowed from the nearest pattern, which is then based on the nearest patients in terms of dropout time and perhaps prognosis and quality of life evolution. However, recall that the identification is done sequentially, and hence even under NCMV, the parameter estimates for pattern 1 are identified borrowing from the remaining patterns 
chronologically.

\subsection{Model Reduction}

As in Section 5.1 we decided to define the covariance structure allowing a separate autoregressive structure per pattern and a residual component common to all patterns. The mean model was initially defined using the baseline covariates and including all interaction terms between time, treatment and pattern. Table 2 displays the F-tests obtained during model simplification. The CCMV and NCMV identifying strategies resulted in the same final model. The time by pattern interaction was not significant in the ACMV model. i.e., yielding the same final model as was found in Section 5.1. As stated before, the ACMV restriction extrapolated patterns predominantly indicating a constant mean score over time thus reducing the interaction between time and pattern.

Although all the restrictions did not yield the same final model we prefer to show the parameter estimates based on the model including the time by pattern interaction. The final parameter estimates for the time effects for this model are presented in Figure 4 . The remaining parameter estimates are presented in Table 3.

The treatment effect is significant at the 0.05 level of significance in favour of prednisone for both the CCMV $(\mathrm{P}=0.011)$ and $\operatorname{ACMV~}(\mathrm{P}=0.027)$ models. However, using the NCMV strategy the treatment effect is no longer significant $(\mathrm{P}=0.123)$. Thus, as the identifying restriction shifts from one extreme (CCMV) to another (NCMV) there is an associated shift in treatment significance. 


\section{Discussion}

Scnsitivity analysis for pattern-mixture models can be conccived in many differcnt ways. Crucial guiding questions are whether a pattern-mixture model is the central focus of interest or should rather be viewed as complementary to another model, such as a selection model. In this paper, we focused on the unidentified components of the pattern-mixture model and illustrated three distinct strategies to fit pattern-mixture models. By contrasting these strategies on a single set of data, one obtains a range of conclusions rather than a single one, which provides insight into the sensitivity to the assumptions made. Especially with identifying restrictions, one has to be very explicit about the assumptions and moreover this approach offers the possibility to consider several forms of restrictions. Special attention should go to the ACMV restrictions, since they are the MAR counterpart within the patternmixture context.

The identifying restrictions strategy provides further opportunity for sensitivity analysis, beyond what has been presented here. Indeed, since CCMV and NCMV are extremes, it is very natural to consider the idea of ranges in the allowable space of $\boldsymbol{\omega}_{s}$. Clearly, any $\boldsymbol{\omega}_{s}$ which consists of non-negative elements that sum to one is allowable, but also the idea of extrapolation could be useful, where negative components are allowed, given they provide valid conditional densities.

Hogan and Laird (1997) noted that in order to estimate the large number of parameters in general pattern-mixture models, one has to make the awkward requirement that each dropout pattern occurs sufficiently often. In other words, one has to require large amounts of dropout or similarly sufficient data in each pattern to estimate the conditional regression from which imputations are calculated.

In the 'imputation' step we included only baseline covariates and an interaction between time 
and treatment term. The model used for imputing the missing values will generally differ from the model used in the analysis. The primary objective in the 'imputation' model is to incorporate enough information in the model to ensure unbiased estimates of the missing values. For example, the reasons for dropout (including progression of disease, treatment toxicity or patient refusal) and time dependent covariates such as performance status, disease status, weight loss, cumulative dose and treatment toxicity may be included in the 'imputation' model. Of course, these factors should not be included in the 'analysis' model as they are factors which are influenced by treatment and may confound the treatment comparisons.

\section{Acknowledgement}

We gratefully acknowledge support from FWO-Vlaanderen Research Project "Sensitivity Analysis for Incomplete and Coarse Data".

\section{References}

[1] Aaronson, N.K., Ahmedzai, S., Bergman, B., et Al. The European Organization for Research and Treatment of Cancer QLQ-C30: a quality-of-life instrument for use in international clinical trials in oncology, Journal of the National Cancer Institute, (1993), 85, 365-376.

[2] Curran, D., Bacchi, M., Schmitz, F. H., Molenberghs, G., and Sylvester, R.J., Identifying the types of missingness in quality of life data from clinical trials, Statistics in Medicine, (1998), 17, 739-756. 
[3] Curran D., Momfnemgras G., Aaronson N., Fossa S. D., Sylufester R. J. Analysis of longitudinal quality of life data with dropout. Statistical Methods in Medical Research, (2002), 11, 5-23.

[4] Diggle, P.J. And Kenward M.G., Informative drop-out in longitudinal data analysis (with discussion) Applied Statistics, (1994), 43, 49-93.

[5] Fairclough, D.L., Peterson, H.F., Cella, D., Bonomi, P., Comparison of several model-based methods for analysing incomplete quality of life data in cancer clinical trials, life in a clinical trial of cancer therapy, Statistics in Medicine, (1998), 17, 781-796.

[6] Fayers, P.M., Aaronson, N.K., Buordal, K., Curran D., And Groenvold, M., EORTC QLQ-C30 Scoring Manual, EORTC, Brussels (1999).

[7] Fossa S.D., Slee P.H.TH, Brausi M., Horenblas S., Hall R.R., Hetherington J.W., Aaronson N., De Prijck L., and Collette L., Flutamide versus prednisone in patients with prostate cancer symptomatically progressing after androgen ablative therapy: a phase III study of the European organisation for research and treatment of cancer genitourinary group. Journal of Clinical Oncology, (2001), 19, 62-71.

[8] Glynn, R.J., Laird, N.M., And Rubin D.B., Selection modelling versus mixture modelling with nonignorable nonresponse, in Wainer, H. (ed. ), Drawing inferences from self selected samples, Springer Verlag, New York, (1986), 115-142.

[9] Hogan, J.W. and Laird, N.M. Mixture models for the joint distribution of repeated measures and event times, Statistics in Medicine, (1997), 16, 239-257.

[10] Hopwood, P., Stephens, R. J., And Machin, D. Approaches to the analysis of quality of life data: experiences gained from a Medical Research Council Lung Cancer Working Party. Quality of Life Research, (1994), 3, 339 - 352. 
[11] Kenward, M.G., Molfmnbrghis, G., and Thi.js, H. Pattern-mixture models with proper time dependence. to Biometrika, (2003) 00, 000-000.

[12] Little, R.J.A. Pattern-mixture models for multivariate incomplete data. Journal of the American Statistical Association (1993), 88, 125-134.

[13] Littrf, R.J.A. A class of pattern-mixture models for normal incomplete data. Biometrika, (1994), 81, $471-483$.

[14] Little, R.J.A., Modeling the drop-out mechanism in repeated-measures studies, Journal of the Americal Statistical Society, (1995), 90, 1112-1121.

[15] Iıtтt.f, R.J.A. And Rubin, D.B., Statistical analysis with missing data, New York: John Wiley, (1987).

[16] Michiels, B., Molenberghs, G., And Lipsitz, S. R. Selection models and patternmixture models for incomplete categorical data with covariates. Biometrics, (1999), 55, $000-000$.

[17] Michiels B, Molenberghs G, Bijnens L, Vangeneugden T, Thijs H. Selection models and pattern-mixture models to analyze longitudinal quality of life data subject to drop-out. Statistics in Medicine, (1998), 21, 1023 - 1041.

[18] Molenberghs G., Lesaffre E. Marginal modelling of correlated ordinal data using an n-way Plackett distribution, Journal of the American Statistical Association, (1994), $89,633-644$.

[19] Molenberghs, G., Michiels, B., And Kenward, M. G. Pseudo-likelihood for combined selection and pattern-mixture models for missing data problems. Biometrical Journal, (1998), 40, $557-572$.

[20] Rubin, D.B., Inference and missing data, Biometrika, (1976), 63, 581-592. 
[21] Rubin, D. B. Multiple Imputation for Nonresponse in Surveys. New York, John Wiley \& Sons, (1987).

[22] Sheiner, L. B., Beale, S. L., And Dunne, A. Analysis of nonrandomly censored ordered categorical longitudinal data from analgesic trials. Journal of the American Statistical Association, (1997), 92, 1235 - 1244.

[23] Thiss, H., Molenberghs, G., Michiels, B., Verbeke, G., and Curran, D. Strategies to fit pattern-mixture models. Biostatistics, (2002) 3, 245-265. 


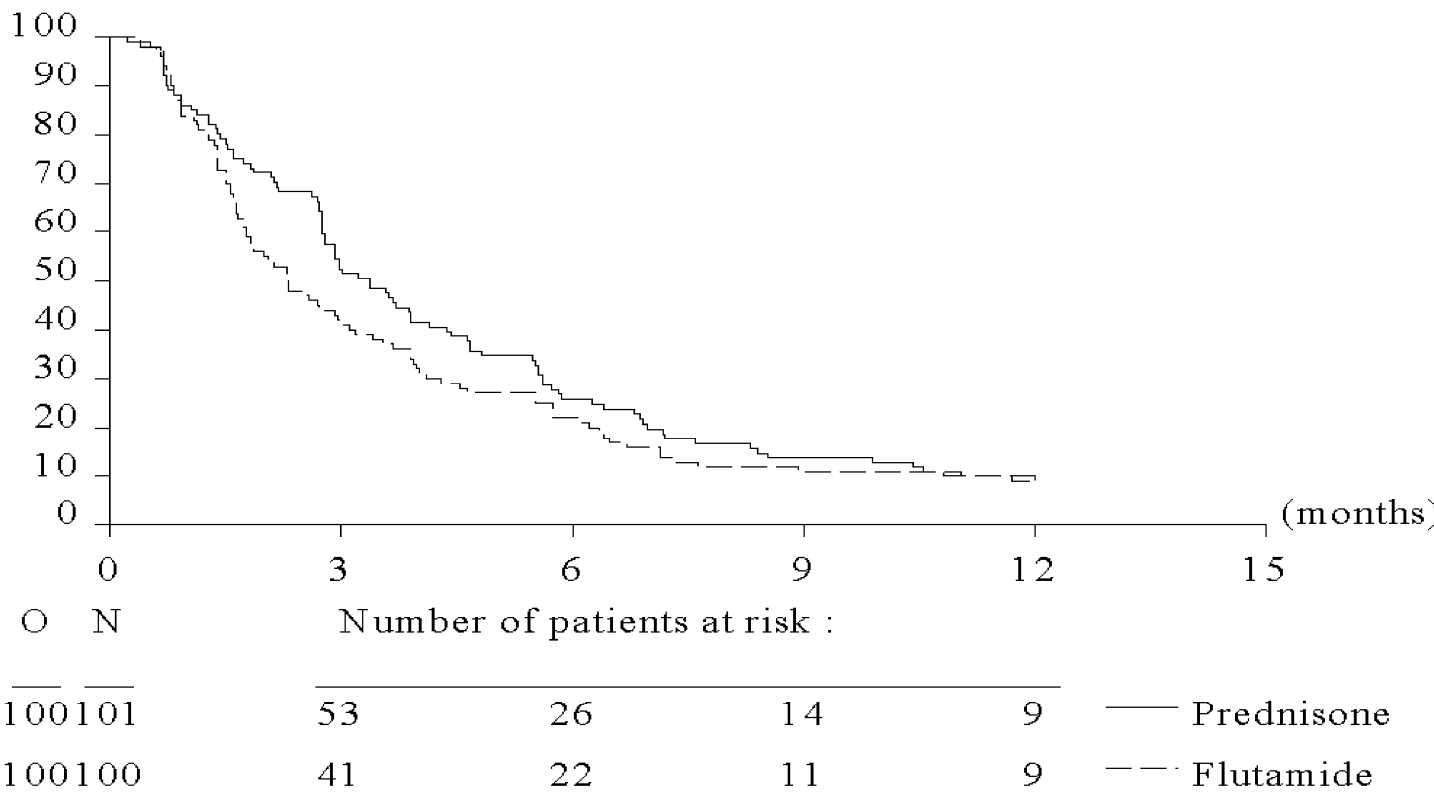

Figure 1: Progression-free survival and associated compliance by treatment arm.

\begin{tabular}{lllllll}
\hline \hline Prednisone \\
\hline Schedule & 0 & 3 & 6 & 12 & 18 & 24 \\
\hline Expected & 101 & 97 & 83 & 65 & 42 & 34 \\
Received & 92 & 77 & 57 & 41 & 29 & 25 \\
$\%$ & 91 & 79 & 69 & 63 & 69 & 73 \\
\hline \hline \multicolumn{7}{l}{} \\
\hline \hline Flutamide & & & & & & \\
\hline Schedule & 0 & 3 & 6 & 12 & 18 & 24 \\
\hline Expected & 100 & 97 & 78 & 44 & 30 & 27 \\
Received & 89 & 73 & 60 & 32 & 21 & 19 \\
$\%$ & 89 & 75 & 77 & 73 & 70 & 70 \\
\hline
\end{tabular}



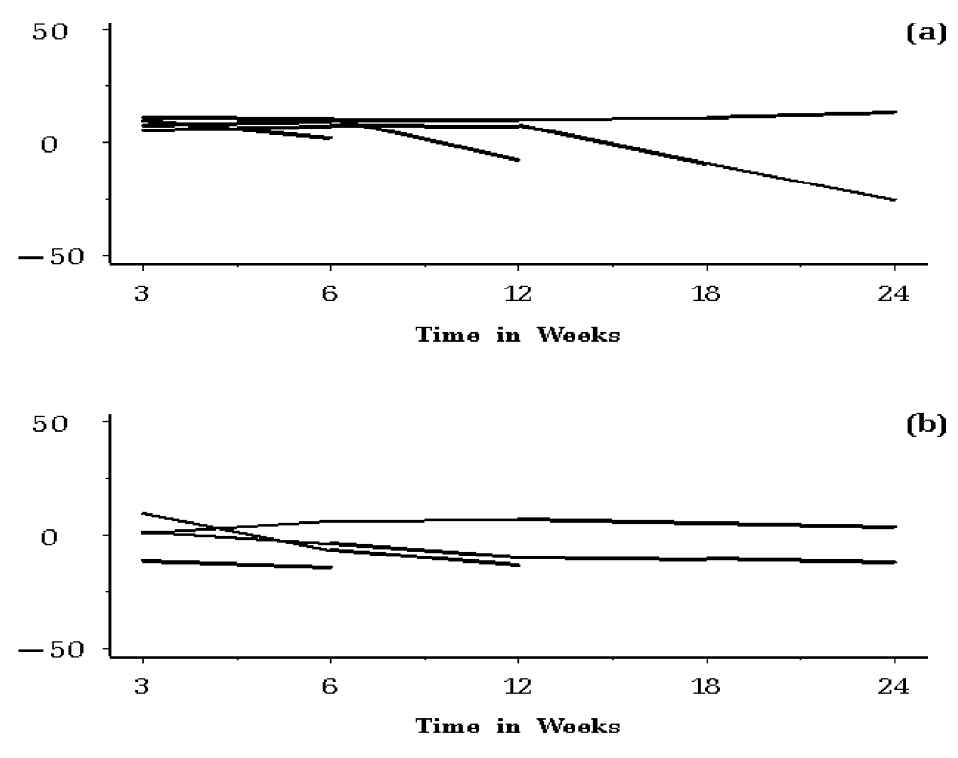

Figure 2: EORTC Trial 30903. Mean profiles by dropout pattern and treatment a) prednisone b) flutamide. 

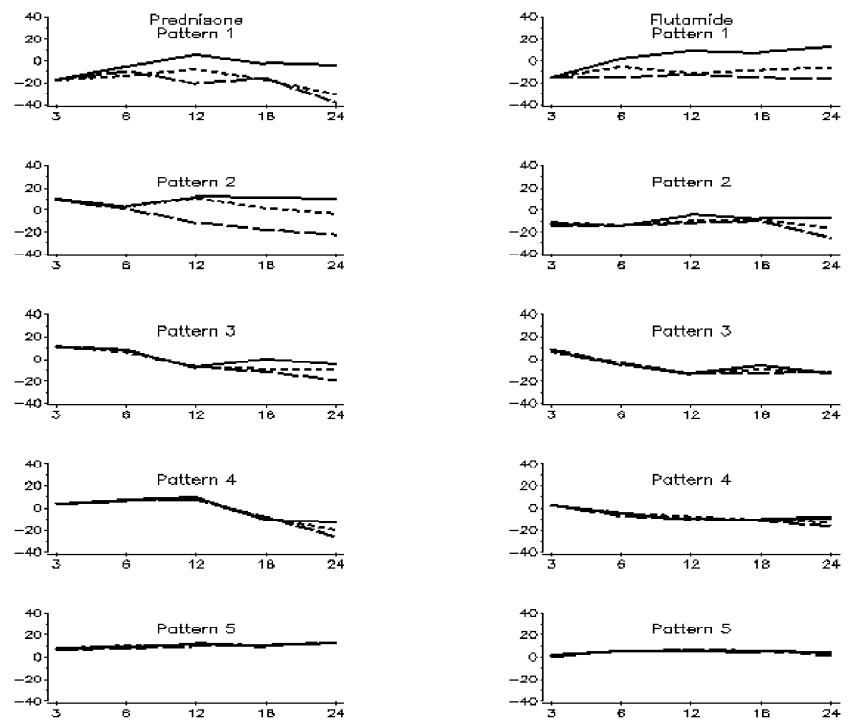

Figure 3: EORTC Trial 30903. Mean response profiles for the multiply imputed datasets using the identifying restrictions CCMV, ACMV and NCMV. Solid line: CCMV, Dotted line: $A C M V$, Dashed line: $N C M V$ 

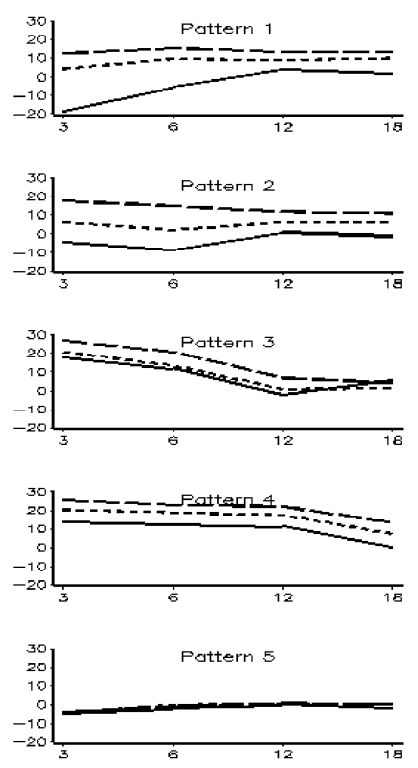

Figure 4: EORTC Trial 30903. Parameter estimates for the time effects for all three patterns, presented by way of profiles over time, for each of the identifying restrictions $C C M V, A C M V$ and NCMV. Solid line: CCMV, Dotted line: ACMV, Dashed line: NCMV 
Table 1: Patterns of missing data.

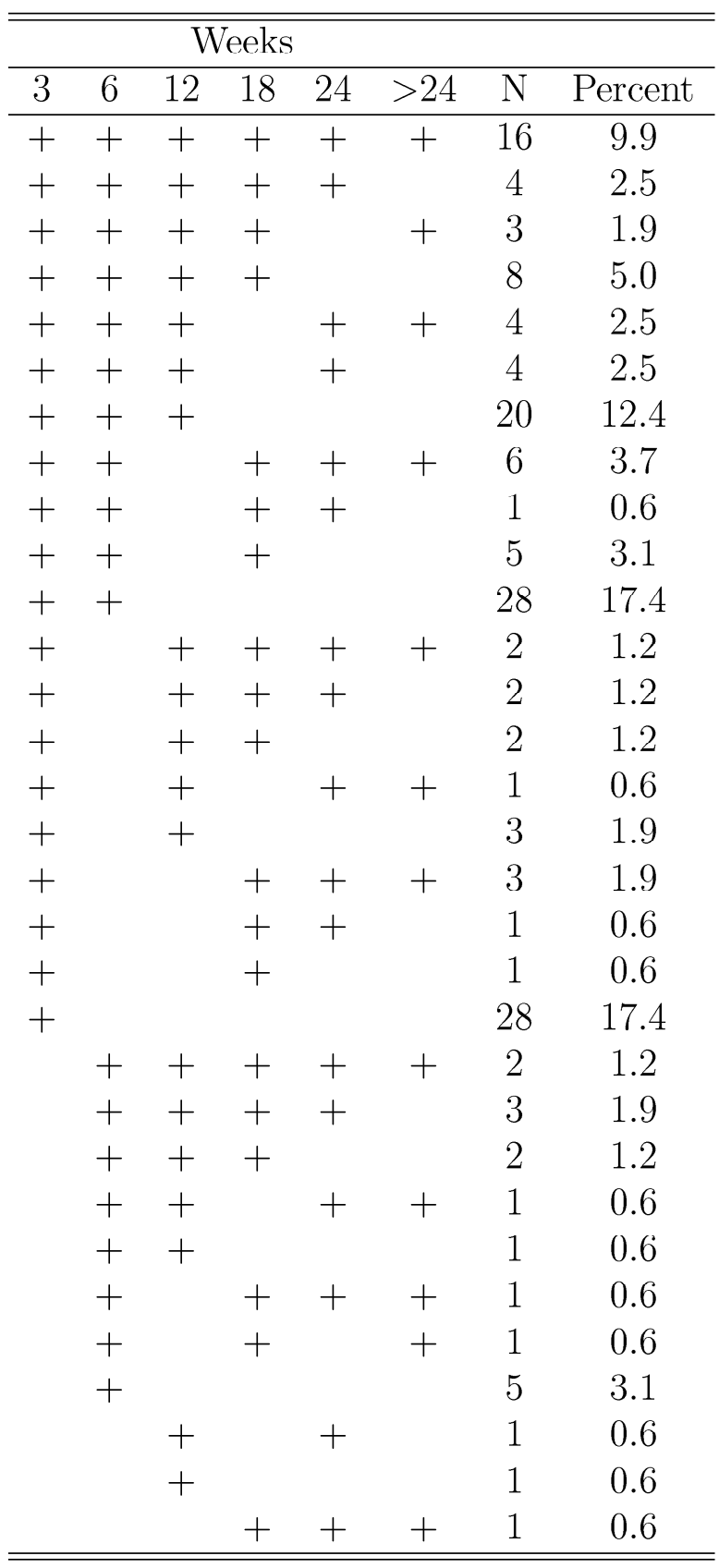


Table 2: EORTC Trial 30903. F-tests for multiple imputation estimates for CCMV, NCMV, and $A C M V$ restrictions. Apart from the $F$ test and $p$ values, numerator and denominator degrees of freedom are shown.

\begin{tabular}{llrrrr}
\hline \hline Model & Effect & $F$ statistic & n.d.f. & d.d.f. & $p$ value \\
\hline \multirow{2}{*}{ CCMV } & & & & & \\
I & 3-way interaction & 0.760 & 16 & 406.6 & 0.731 \\
II & treatment*pattern & 0.886 & 4 & 438.8 & 0.472 \\
III & treatment*time & 0.598 & 4 & 50.7 & 0.666 \\
IV & time*pattern & 2.590 & 16 & 266.2 & 0.001 \\
& & & & & \\
ACMV & & & & & \\
I & 3-way interaction & 0.671 & 16 & 513.8 & 0.824 \\
II & treatment*pattern & 0.712 & 4 & 904.7 & 0.584 \\
III & treatment*time & 1.241 & 4 & 305.5 & 0.293 \\
IV & time*pattern & 1.591 & 16 & 275.4 & 0.070 \\
& & & & & \\
NCMV & & & & & \\
I & 3-way interaction & 1.461 & 16 & 601.1 & 0.109 \\
II & treatment*pattern & 0.486 & 4 & 771.5 & 0.746 \\
III & treatment*time & 0.578 & 4 & 56.8 & 0.680 \\
IV & time*pattern & 2.589 & 16 & 599.8 & 0.001 \\
\hline \hline
\end{tabular}


Table 3: EORTC Trial 30903. Multiple imputation parameter estimates using CCMV, $N C M V$, and $A C M V$ restrictions, excluding time by pattern effects which are graphically presented in Figure 4.

\begin{tabular}{|c|c|c|c|c|c|c|c|c|c|}
\hline \multirow[b]{2}{*}{ Effect } & \multicolumn{3}{|c|}{$\overline{\mathrm{CCMV}}$} & \multicolumn{3}{|c|}{$\overline{\mathrm{ACMV}}$} & \multicolumn{3}{|c|}{ NCMV } \\
\hline & Est. & s.e. & $p$ & Est. & s.e. & $p$ & Est. & s.e. & $p$ \\
\hline \multicolumn{10}{|l|}{ Mean Structure Parameters: } \\
\hline Intercept & 15.3 & 14.0 & 0.274 & 14.5 & 13.8 & 0.294 & 3.6 & 12.9 & 0.783 \\
\hline Age & -0.6 & 3.0 & 0.830 & -1.7 & 3.1 & 0.570 & -4.4 & 2.8 & 0.118 \\
\hline WHO Category 1 & 4.2 & 4.3 & 0.331 & 4.8 & 4.3 & 0.264 & 4.9 & 4.2 & 0.245 \\
\hline WHO Category 2 & 7.4 & 5.3 & 0.161 & 6.4 & 5.7 & 0.264 & 2.8 & 5.9 & 0.637 \\
\hline WHO Category 3 & 9.9 & 8.2 & 0.227 & 8.9 & 8.0 & 0.264 & 8.8 & 8.0 & 0.275 \\
\hline Pain Category 0 & -20.2 & 13.7 & 0.141 & -17.7 & 14.4 & 0.221 & -3.4 & 12.8 & 0.789 \\
\hline Pain Category 1 & -17.5 & 13.2 & 0.185 & -17.2 & 12.8 & 0.179 & -2.2 & 11.0 & 0.839 \\
\hline Pain Category 2 & -10.9 & 12.8 & 0.397 & -9.7 & 13.1 & 0.460 & 0.9 & 11.6 & 0.936 \\
\hline Pain Category 4 & -10.9 & 13.1 & 0.408 & -10.6 & 12.7 & 0.404 & 3.6 & 11.8 & 0.760 \\
\hline Treatment Effect & 7.6 & 3.0 & 0.011 & 6.6 & 3.0 & 0.027 & 4.8 & 3.0 & 0.123 \\
\hline Pattern 1 & -8.2 & 8.8 & 0.349 & -29.8 & 12.5 & 0.032 & -36.2 & 9.6 & $<0.001$ \\
\hline Pattern 2 & -9.2 & 5.8 & 0.114 & -18.8 & 7.7 & 0.024 & -30.8 & 8.0 & 0.001 \\
\hline Pattern 3 & -18.7 & 7.3 & 0.014 & -20.7 & 7.5 & 0.008 & -26.8 & 7.6 & 0.001 \\
\hline Pattern 4 & -20.4 & 5.3 & $<0.001$ & -25.8 & 5.6 & $<0.001$ & -29.5 & 5.3 & $<0.001$ \\
\hline \multicolumn{10}{|l|}{ Variance Components: } \\
\hline $\operatorname{AR}(1)$ Varia & 599.8 & 230.9 & 0.016 & 643.7 & 253.1 & 0.024 & 444.4 & 155.4 & 0.015 \\
\hline AR(1) Corr. for Patt. 1 & 0.9 & 0.1 & 0.001 & 0.8 & 0.1 & $<0.001$ & 0.2 & 0.4 & 0.567 \\
\hline AR(1) Variance for Patt. 2 & 280.2 & 137.4 & 0.077 & 380.9 & 134.4 & 0.018 & 373.6 & 83.2 & $<0.001$ \\
\hline AR(1) Corr. for Patt. 2 & 0.8 & 0.2 & 0.010 & 0.7 & 0.2 & 0.008 & 0.4 & 0.2 & 0.041 \\
\hline AR(1) Variance for Patt. 3 & 322.8 & 134.4 & 0.031 & 393.7 & 136.9 & 0.015 & 312.7 & 78.3 & $<0.001$ \\
\hline $\operatorname{AR}(1)$ Corr. for Patt. 3 & 0.8 & 0.2 & 0.021 & 0.6 & 0.2 & 0.036 & 0.5 & 0.1 & $<0.001$ \\
\hline AR(1) Variance for Patt. 4 & 354.6 & 107.2 & 0.002 & 362.9 & 116.9 & 0.007 & 309.9 & 88.7 & 0.001 \\
\hline AR(1) Corr. for Patt. 4 & 0.9 & 0.2 & 0.004 & 0.8 & 0.2 & 0.013 & 0.8 & 0.1 & $<0.001$ \\
\hline AR(1) Variance for Patt. 5 & 311.5 & 109.6 & 0.014 & 344.9 & 90.6 & 0.001 & 333.4 & 82.2 & $<0.001$ \\
\hline AR(1) Corr. for Patt. 5 & 0.9 & 0.2 & 0.003 & 0.9 & 0.2 & 0.007 & 1.0 & 0.1 & $<0.001$ \\
\hline Common meas. error var. & 128.2 & 79.6 & 0.178 & 90.4 & 90.6 & 0.373 & 119.3 & 24.5 & $<0.001$ \\
\hline
\end{tabular}

